\title{
INTRODUCTION OF CARRIER INTERFERENCE TO SPREAD SPECTRUM MULTIPLE ACCESS
}

\author{
Carl R.Nassar $\uparrow$. Balasubramaniam Natarajan $\dagger$, Steve Shattil $\ddagger$ \\ † Department of ECE \\ Colorado State University \\ Fort Collins, CO 80523-1373 \\ carin@engr.colostate.edu \\ $\ddagger$ Idris Communications \\ 4980 Meredith Way \# 201 \\ Boulder, CO 80303 \\ temporal@dimensional.com
}

nbalsu@engr.colostate.edu

\begin{abstract}
This paper introduces a new scheme for spread spectrum multiple access. Like MCCDMIA, this scheme accomplishes spectral spreading by transmission of identical data over $\mathrm{N}$ carriers simultaneously. However, unlike any existing CDMA technique to date, this method supports user orthogonality not through the use of spreading rodes (based on $\mathrm{PN}$ sequences), but rather through multiple carrier interference. Specifically, in this novel method, aptly named CIMA (Carrier Interference Multiple Access), the interference of multiple carriers enables user orthogonality or pseudoorthogonality based on user positioning in time. It is shown that CIMA supports simplified receiver structures for AWGN channels. and offers performance benefits in AWGN and fading environments.
\end{abstract}

\section{INTRODUCTION}

Direct sequence code division multiple access (DSCDMA) has emerged as a popular multiple access scheme in commercial applications. The wide bandwidth of DS-CDMA signals results in frequency selective multipath fading which, when combined with a RAKE receiver, supports path diversity gains. However, the path diversity benefits are only attainable when continuous estimation of path gain and path delay are implemented. This demands significant signal processing power, especially in the pres'nce of other user's multipath interference. A second conrern in DS-CDMA arises in high data rate appli(itions, when channel delay spread exceeds the data symbol duration. In such cases DS-CDMA is subject to serere ISI(Inter-symbol Interference) and ICI
(Inter chip Interference).

Recently, the promise of OFDM (orthogonal frequency division multiplexing) has been successfully combined with CDMA, resulting in the introduction of multi-carrier CDMA (MC-CDMA)[1]. Here, each data symbol is transmitted simultaneously over $\mathrm{N}$ narrowband subcarriers, with each subcarrier encoded with the $-\pi$ or $\pi$ phase offset (as determined by a PN code sequence). Multiple access is supported by assigning different users (which transmit over the same subcarriers) different PN codes - orthogonal to the codes of other users.

MC-CDMA achieves significant performance benefits over conventional DS-CDMA. These result because diversity gains stem from frequency diversity rather than path diversity, allowing for improved combining without loss due to ICI [2].Furthermore, in high data rate applications, MC-CDMA is capable of spreading the signal bandwidth while avoiding the adverse effect of the delay spread on the original data [2].

This paper introduces a new spread spectrum multiple access method known as Carrier Interference Multiple Access (CIMA). CIMA is similar to MCCDMA in that identical data symbols are sent over $\mathrm{N}$ subcarriers simultaneously. However, multiple access in CIMA is attained not through the use of $P N$ sequences but rather through a novel carrier interference scheme. CINIA will be shown to demonstrate a number of benefits relative to MC-CDMA. Firstly, MC-CDMA may utilize either orthogonal or pseudo orthogonal codes. Once a selection is made, no changes are possible in the MC-CDMA case. In CIMA systems, orthogonality among users is always attained wherever the number of users is less than or equal to the number of dimensions (number of 
carriers), and pseudo orthogonality among users is achieved once the number of uscrs exceeds the number of carriers. That is, CIMIA systems naturally su'itch from orthogonality to pseudo orthogonality, and back again as dictated by the number of users. Additionally, in AlYGN (Additive White Gaussian Noise) channels, CIMA supports a greatly simplified receiver structure. CIMA also promises a number of additional benefits which will be discussed at the end of this work.

This paper is organized as follows: Section 2 introduces the CIMA signal and its characteristics. Sections 3,4 and 5 present the CIMA transmitter, the channel model and the CIMA receivers respectively. Section 6 introduces the performance of CIMA. Throughout. BPSK modulation is utilized to simplify the presentation of the new system, but other modulation techniques are equally applicable.

\section{CIMA SIGNALING}

The hasic (IMlt signal corresponds to the superpositioning of $X$ carriers equally spaced in frequency hy $\Delta f$. The cumulative signal corresponds to

$$
r(t)=\sum_{i=0}^{Y-1} \cos \left(\left(2 \pi f_{t}+i 2 \pi \Delta f\right) t\right) .
$$

as shuwn in Figure 1 with $Y=16$. This signal corresponds to a cosine waveform with frequency $f_{1}+\frac{|x-1|}{2}>f$ and an envelope given by

$$
E(t)=\left|\frac{\sin \left(\frac{1}{2} \Lambda^{2} 2 \pi \Delta f t\right)}{\sin \left(\frac{1}{2} 2 \pi \Delta f t\right)}\right|
$$

Figure 2 shows the envelope of the CIMA signal for $. X=16$ carriers. CIMA envelopes are periodic with period $1 / \Delta f$. Within each period, mainlobes demonstrate a duration of $\frac{2}{N S f}$ and the $N-1$ sidelobes a duration of $\frac{1}{N j f}$. The $l^{\text {th }}$ side lobe has maximum amplitude (normalized with respect to mainlobe anplitude)

$$
A(l)=\frac{1}{N \sin \frac{\pi}{N}\left(l+\frac{1}{2}\right)}
$$

By introducing a phase offset to each subcarrier, spercifically the offset $i \Delta \theta$ to the $i^{\text {th }}$ subcarrier, the "nvelope of the CIMA signal is shifted in time by $\Delta t=\frac{\Delta \theta}{2 \pi \Delta f}$. A rareful selection of $\Delta \theta$ leads to orthegenality in time betwern the shifted and the unshifted (IMA signal. as shown in Figure 3. In this way, two user's CIMA signals can be positioned orthogonally.

The CIMA signal employed by a second user has an envelope corresponding to a time shifted version of the CIMA envelope of a first user. The expression for $\mathrm{CC}$ between user $\mathrm{k}$ and user $\mathrm{j}$, with a time shift between envelopes of $\tau$, can be shown to be

$$
\begin{gathered}
R_{k, j}(\tau)=\frac{1}{2 \Delta f} \sum_{i=0}^{N-1} \cos (i(2 \pi \Delta f \tau)) \\
R_{k, j}(\tau)=\frac{1}{2 \Delta f} \cdot \frac{\sin \left(\frac{1}{2} N 2 \pi \Delta f \tau\right)}{\sin \left(\frac{1}{2} 2 \pi \Delta f \tau\right)} \cdot \cos \left(\frac{(N-1)}{2} 2 \pi \Delta f \tau\right)
\end{gathered}
$$

This CC term demonstrates $2(N-1)$ zeros:

- $N-1$ equally spaced zeros at $\left\{\frac{k}{N \Delta f}, k=\right.$ $1,2, \ldots, N-1\}$ as a result of the $\frac{\sin (\cdot)}{\sin (\cdot)}$ term and

- $N-1$ equally spaced zeros at $\left\{\frac{2 k-1}{2(N-1) \Delta f}, k=\right.$ $1,2, \ldots, N-1\}$ as a result of the $\cos (\cdot)$ term.

This indicates that there exist $2(N-1)$ locations in time where a second user may be placed (by choice of $\Delta \theta$ ) to maintain orthogonality with respect to a first user.

The existence of one set of $N-1$ equally spaced zeros indicates that a CIMA system can simultaneously support $N$ orthogonal users. The existance of a second set of zeros indicates that we can place the users orthogonally at either (1) positions corresponding to the first set of zeros, or (2) at positions corresponding to the second set of zeros.

The spacing between the first set of $N-1$ zeros and the second set of $N-1$ zeros is such that users positioned in one set of zeros are nearly orthogonal (pseudo orthogonal) to users positioned at the other set of zeros. Hence, a CIMA system can support $\mathrm{N}$ orthogonal users, and, additionally, if more users are to be accommodated, it can support these users pseudo orthogonally by placing them on the second set of zeros.

\section{TRANSMITTER MODEL}

The transmitter for the $k^{\text {th }}$ user in a CIMA system is shown in Figure 4. The input data symbol, $a_{k}[\mathrm{n}]$, is assumed to be binary antipodal where $n$ denotes the $n^{\text {th }}$ bit interval and $k$ denotes the $k^{\text {th }}$ user. It is assumed that $a_{k}[\mathrm{n}]$ takes on values -1 and +1 with equal probability. The transmitted signal 
corresponding to the $n^{\text {th }}$ data bit of the $k^{t h}$ user is

$$
s_{k}(t)=\sum_{i=0}^{N-1} a_{k}[n] \cos \left(2 \pi f_{1} t+i ذ \theta_{k}\right)
$$

where $f_{1}=f_{t}+i \pm f$

The total transmitted signal considering all users and assuming downlink transmission is

$$
s(t)=\sum_{k=1}^{k} \sum_{i=0}^{N-1} a_{k}[n] \cos \left(2 \pi f_{i} t+i \Delta \theta_{k}\right)
$$

where $\mathrm{h}$ is the total number of users in the system. In a CIMIA system the $\Delta f$ 's are selected such that the carrier frequencies $\left\{f_{i}, i=0,1, \ldots, N-1\right\}$, are orthogonal to each other. This aids in synchronization at the receiver. It can be shown that the rhoice of minimum $\perp f$ that ensures orthogonality is $\Delta f=1 / T_{b}$. where $T_{b}$ is the bit duration.

\section{CHANNEL MODEL}

T1 simplify our presentation. we assume downlink communication throughout this paper.

Wo first address a simple AllG channel, modeling the ideal power cont rol scenario in wireless links. In this atse.

$$
r(t)=s(t)+n(t)
$$

where $n(t)$ demotes the AWG.N with power spectral density $\lambda_{0} / 2$

Serondly, we consider a slowly varying frequencyselective Rayleigh fading channel. Frequency selectivity refers to the selectivity over the entire bandwidth of transmission. and not orer each of the subcarrie'r transmissions: that is

$$
1 / T_{b}<<(\Delta f)_{c}<B H^{\circ}
$$

where $(\Delta f)$, is the coherence bandwidth and $B W$ is the total bandwidth of the multicarrier system [3]. In this work. we examine frequency selectivity resulting in two fold frequency diversity over the entire bandwidth (as in [2]). While in some works this is represented as $(\Delta f)_{c} / B H^{\circ}=0.50$ [4], we have foumd that a hetter representation corresponds to $(\Delta f) / B H^{\circ}=0.25[3]$.

With 1 carricers residing over the entire bandwidth, BII. each carrier undergoes a flat fade, with the correlition betwern the $i^{\text {th }}$ subcarrier fade and the $j^{\prime \prime \prime}$ subratrion fatde characterized by [4]

$$
\rho_{1 . j}=\frac{1}{1+\left(\left(f_{1}-f_{\jmath}\right) /(\Delta f)_{i}\right)^{2}}
$$

where $\left(f_{i}-f_{j}\right)$ indicates the frequency separation between the $i^{t h}$ and the $j^{\text {th }}$ subcarriers. Generation of two fades with correlation has been discussed in [5], and a more general method is presented in [6].

\section{RECEIVER STRUCTURES}

The received signal is characterized by

$r(t)=\sum_{k=1}^{K} \sum_{i=0}^{N-1} \alpha_{i} a_{k}[n] \cos \left(2 \pi f_{i} t+i \Delta \theta_{k}+\phi_{i}\right)+\eta_{i}(t)$

where $\alpha_{i}$ is the gain and $\phi_{i}$ the phase offset due to the channel; and $\eta_{i}(t)$ represents AWGN. To simplify the analysis, exact phase synchronization is assumed.

The CIMA receiver for user $\mathrm{k}$ is shown in Figure 5. Here, the received signal is projected onto the orthonormal basis of the transmitted signal, outputting $\underline{\underline{r}}=\left(r_{0}, r_{1}, \ldots ., r_{N-1}\right)$ where

$r_{i}=\alpha_{i} a_{k}[n]+\sum_{j=1, j \neq k}^{K} \alpha_{i} a_{j}[n] \cos \left(i\left(\Delta \theta_{k}-\Delta \theta_{j}\right)\right)+\eta_{i}$

where $\eta_{i}$ is a Gaussian random variable with mean 0 and variance $N_{o} / 2$.

Next a suitable combining strategy is used to create a decision variable, $D$ which then enters a decision device with output $\hat{a}_{k}[n]$.

In the case of an AWGN channel $\left(\alpha_{i}=1\right.$ and $\phi_{i}=$ $0)$, CIMA supports the simplified receiver structure shown in Figure 6. Instead of $\mathrm{N}$ bandpass filters, the CIMA receiver employs a single matched filter matched to the $k^{t h}$ user's CIMA envelope (Figure 2).

In fading channels, the general receiver of Figure 5 is employed. Different combining methods may be used, such as the popular EGC (Equal Gain Combining), MRC (Maximal Ratio Combining), ORC (orthogonality restoring combining) and MMSEC (Minimum mean square error combining). MMSEC has been shown to produce the best performances in MC-CDMA [2]. Employing MMSEC in our case results in the decision variable $D$ given by the linear sum

$$
D=\sum_{i=0}^{N-1} r_{i} \cdot\left[\frac{\alpha_{i}}{\left(K \alpha_{i}^{2}+N_{o}\right)}\right]
$$




\section{PERFORMANCE RESULTS}

Figure i presents the bit error rate (BER) versus number of users, when $N=32, S N R=16 \mathrm{~dB}$ and MMSEC combining is employed. Results are presented for a frequency selective Rayleigh fading (hannel with both $(\Delta f)_{c} / B W=0.5$ (Figure 7 ) and $(\Delta f)_{1} / B H^{\circ}=0.25$ (Figure 8). The lower bound is two-fold diversity performance.

Comparison is made with MC-CDMA. Two MCCDNA curves are provided, the first assumes orthogonal Hadamard-Walsh (HW) codes of length 32 (dashed line) and the second assumes gold codes (solid line).

As soen in Figures 7 and 8, CIMA BERs (dotted line) match those of orthogonal MC-CDMA up to 32 usirs. While orthogonal MC-CDMA can not silpport additional users. CIMA is shown to accommodate a growing number of users. If MC-CDMA is pre-selected to support additional users, by use of psoudo orthogonal gold codes, it results in performance degradation as shown by the solid line. ClNA. however, offers the performance of orthogonal MC-CDMA with the flexibility (in terms of number of users) of non-orthogonal MC-CDMA.

Figures $T$ and 8 together show the performance of CIMA and MC-CDMA improving with decrease in $(\Delta f) . / B W$ : i.e. in the cases of a less correlated fading wer the subcarriers. performance improves.

\section{DISCUSSION AND CON- CLUSIONS}

In this paper, C1MA. an imovation in spread spectrum multiple access, is introduced. For the AWGN channel. CIMA facilitates the use of a simplified recoiver structure. In AlWGN and frequency selective Rayleigh fading channels. CIMA's performance natche's that of orthogonal MC-CDMA up to the MC-CDMA $N$ user limit. CIMA provides the added Hexibility of going beyond $\mathrm{N}$ users, by adding users with pseudes orthogonal positioning.

Fortheoming rescarch will focus on demonstrating the additional benefits of a CIMA system. This rescarch will include a study of the following :

- Thr subcarriers which compose a CIMA signal can br assigned amplitude weights to create reduced siblelse activity in the time domain, enabling CIMIA to support multiple users with reduced interference. - CIMA will be combined with FDM to significantly enhance frequency diversity benefits.

- Flexibility of user positioning in time allows for positionings that optimize various criteria, (e.g., minimizing near-far effects, optimizing performance for a given combining/Multiuser Detection (MUD) strategy).

- A merger between CIMA signals and antenna arrays will be explored, and benefits in terms of increased capacity, spatial diversity, and spatial sweeping will be examined.

The benefits of CIMA presented in this work, coupled with those anticipated from forthcoming research, may enable CIMA to achieve notoriety among existing multiple access techniques.

\section{REFERENCES}

[1] N. Yee, J. P. Linnartz and G. Fettweis, "MultiCarrier CDMA in indoor Wireless Radio," in Proc. PIMRC '93, Yokohama, Japan, Dec. 1993, pp. 109 113.

[2] S. Hara and R. Prasad, "Overview of multicarrier CDMA," IEEE Communications Magazine, vol.35, no. 12 , Dec. 1997 , pp. 126-133.

[3] J.Proakis, Digital Communications. New York: McGraw-Hill, $3^{\text {rd }}$ ed., 1995.

[4] W.Xu and L.B.Milstein, "Performance of Multicarrier DS CDMA Systems in the presence of correlated fading," IEEE $47^{\text {th }}$ Vehicular Technology Conference, Phoenix, AZ, May 4-7, 1997, pp. 2050-4

[5] R.B.Ertel and J.H.Reed, "Generation of Two Equal Power Correlated Rayleigh fading envelopes," IEEE Communication Letters, vol.2, no. 10, Oct. 1998, pp. 276-278

[6] B.Natarajan,C.R.Nassar and V.Chandrasekhar, "Generation of N Equal Power Rayleigh fading envelopes," to be submitted to IEEE Communications Letters.

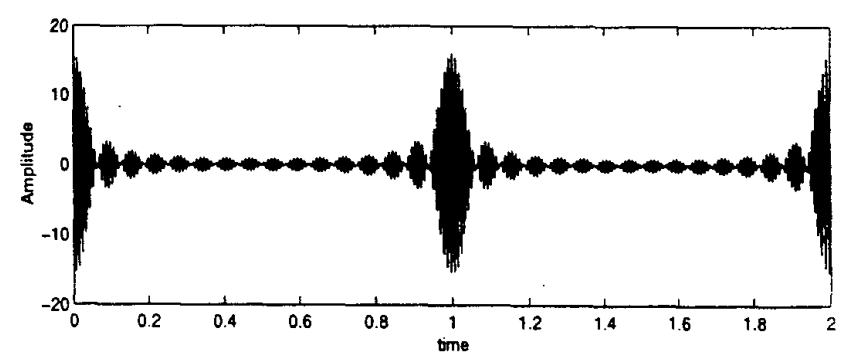

Figure 1: CIMA signal including the carrier 


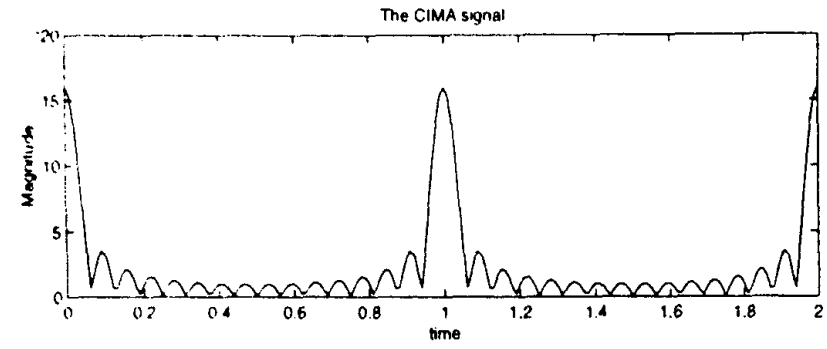

Figure 2: Envelope of a CIMA signal

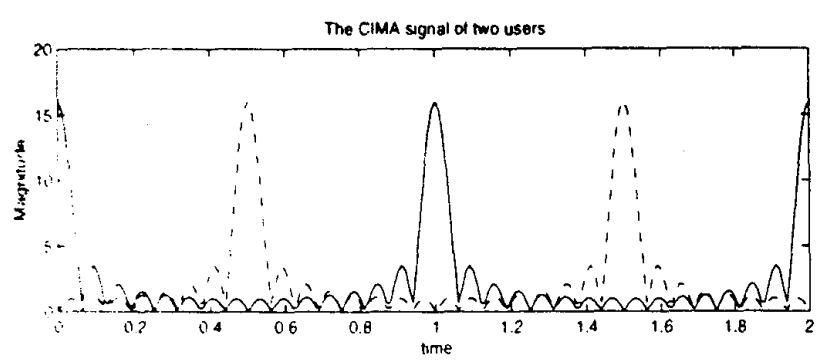

Figure 3: Envelopes of CIMA signals of two users orthogonal in time

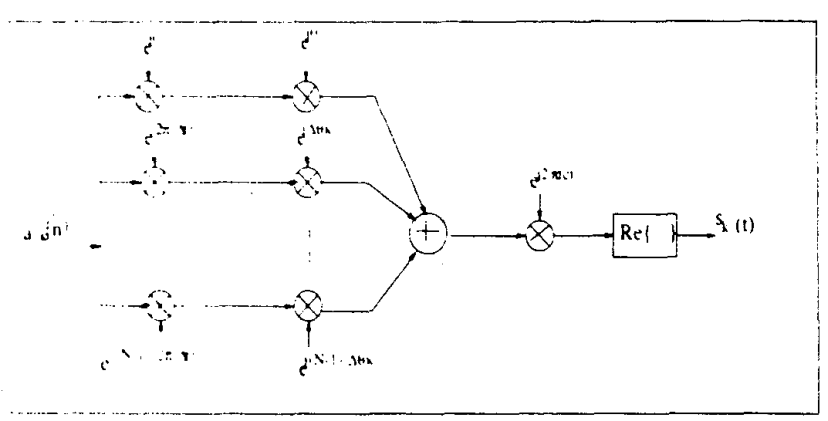

Figure 4: CIMA Transmitter of $k^{\text {th }}$ user

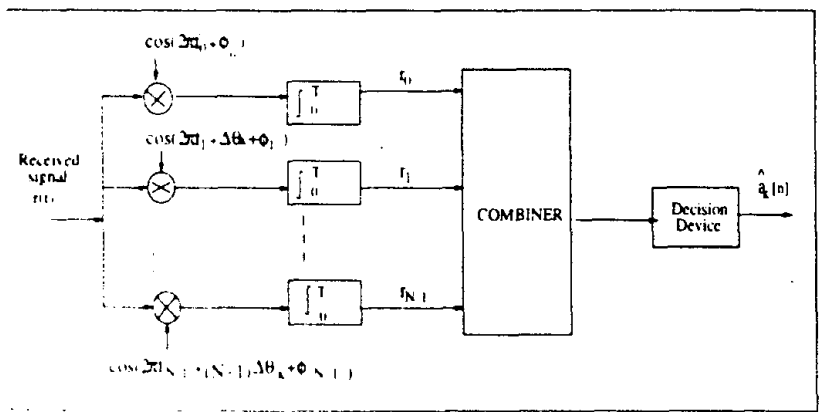

Figure 5: CIMA Receiver

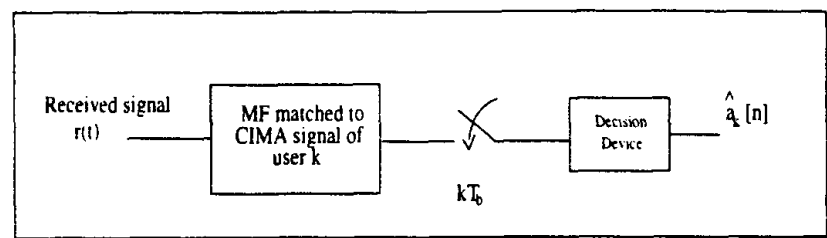

Figure 6: Simplified CIMA Receiver for AWGN channel

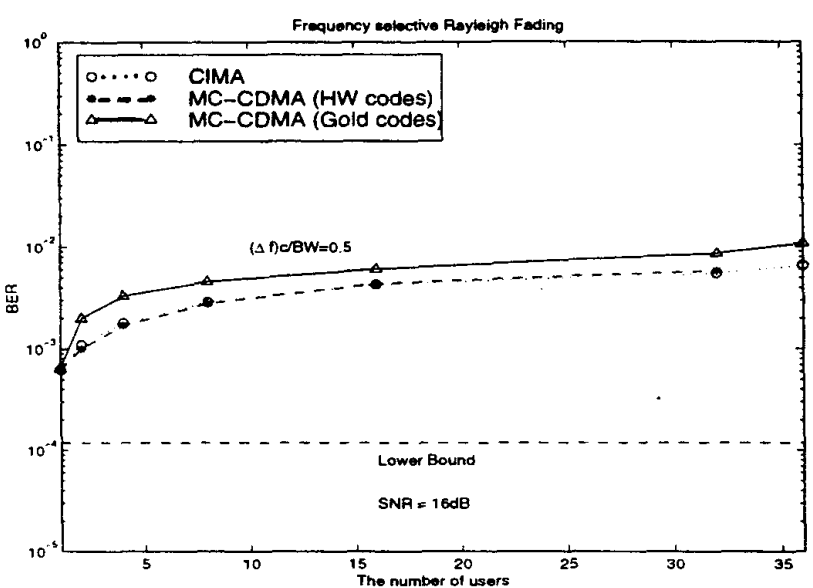

Figure 7: BER performance of CIMA, orthogonal MC-CDMA and pseudo orthogonal MC$\mathrm{CDMA} ;(\Delta f)_{c} / B W=0.5$

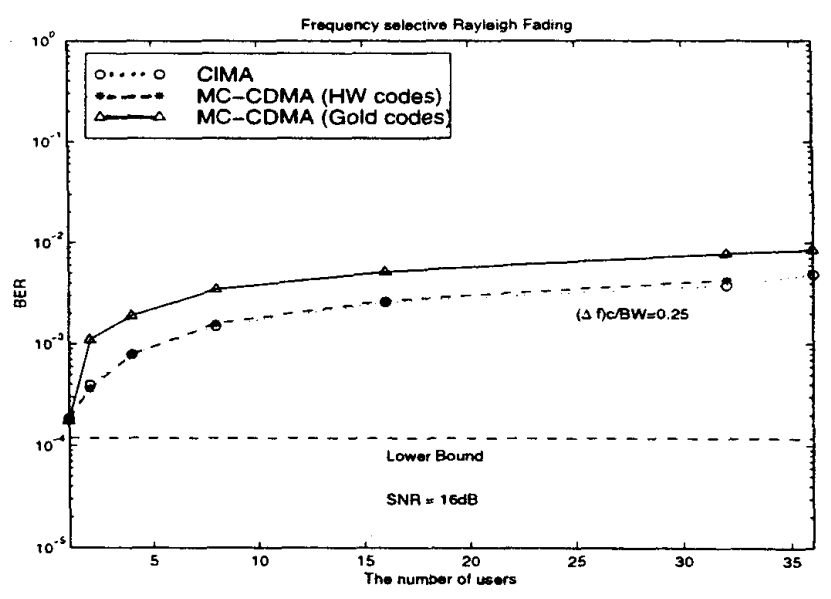

Figure 8: BER performance of CIMA, orthogonal MC-CDMA and pseudo orthogonal MC$\mathrm{CDMA} ;(\Delta f)_{c} / B W=0.25$ 